\title{
Congenital complete absence of left pericardium
}

\author{
Report of two cases
}

\author{
J O H N B O R R I \\ From the Department of Thoracic Surgery, University of Otago Medical School, and \\ Southern Regional Thoracic Surgical Unit, Dunedin, New Zealand
}

Two cases of congenital complete absence of the left pericardium are presented. One, in a youth aged 17 years, had an associated patent ductus arteriosus; the other occurred in a woman aged 65 years with carcinoma of the lower third of the oesophagus. In both, the pericardial lesion was discovered as an incidental finding-at thoracotomy. The anatomical and radiographic features are described. Complete absence of the left side of the pericardium needs no surgical treatment and is compatible with normal life expectancy. On the other hand, because of the dangers of herniation of the heart with strangulation, partial defects should be closed, using either mediastinal pleura or fabric such as Dacron or Teflon.

Congenital absence of the pericardium is rare. In 7,500 necropsies cross-indexed since 1949 in the Department of Pathology, University of Otago, none was recorded, while in 12,000 recorded necropsies since 1921, only one was seen (D'Ath, 1969). The defect may be partial or complete. Though first described by Realdus Columbus in 1559 , and thereafter noted as an unusual necropsy finding (Rusby and Sellors, 1945), it was not until 400 years later, in 1959, that Ellis, Leeds, and Himmelstein (1959) reported the first case where the diagnosis, suggested on the bases of plain chest films, was correctly established pre-operatively by diagnostic pneumothorax.

The purpose of this paper is to describe two patients seen in the space of one year, in whom this anomaly was unexpectedly encountered during left thoracotomy, to show radiographic features that can suggest its presence and to emphasize that complete absence of the left pericardium is a benign condition, associated with normal life expectancy.

\section{CASE REPORTS}

CASE 1 Mr. I. K. McC. (95805), aged 17 years. referred by Dr. Thodey to the Southern Regional Thoracic Surgical Unit, Dunedin, for surgical treatment of patent ductus arteriosus, was admitted to Wakari Hospital on 28 November 1966.

The patent ductus arteriosus had first been detected when he was examined for life insurance five months previously.
He gave no history of effort intolerance, playingo routine sport at school without any limitation of physical activity. His past history added nothing Physically he was obese with a high colour. The radial pulse rate was regular at 72 per minute. Theo femoral pulse was normal, as was the jugular venouø pulse. The blood pressure was $130 / 70 \mathrm{~mm}$. $\mathrm{Hg}$. The apex beat was in the 5 th left intercostal space, $5 \frac{1}{2}$ in $\frac{3}{3}$ $(13.9 \mathrm{~cm}$.) from the mid-sternal line. There was thrill palpable in the 2nd left intercostal space, associated with a harsh continuous machinery murmur maximal at the site of the thrill, and transmitted to wards the left shoulder. No other abnormalities were్ detected. The electrocardiogram showed the heart in sinus rhythm with minimal evidence of left ventriculas hypertrophy. Professor O'Donnell substantiated the clinical diagnosis of patent ductus arteriosus with? moderate left-to-right shunt.

Fluoroscopy (Dr. C. Wiggins) While the overall heart size appeared normal, the unusual contour suggested some left ventricular enlargement, due tô laevorotation, which caused marked prominence of the main pulmonary artery. The peripheral pulmonary vessels were small with no sign of pleonamia. The appearances were consistent with a small patenf ductus arteriosus associated with 'congenital laevo< rotation of the heart' (Fig. 1).

Surgical closure was advised and performed or 5 December 1966 via a left 4 th intercostal postero? lateral thoracotomy.

Operative findings On opening the left pleura cavity it was noted that the lungs showed the usuaf brick-red colour associated with patent ductus 


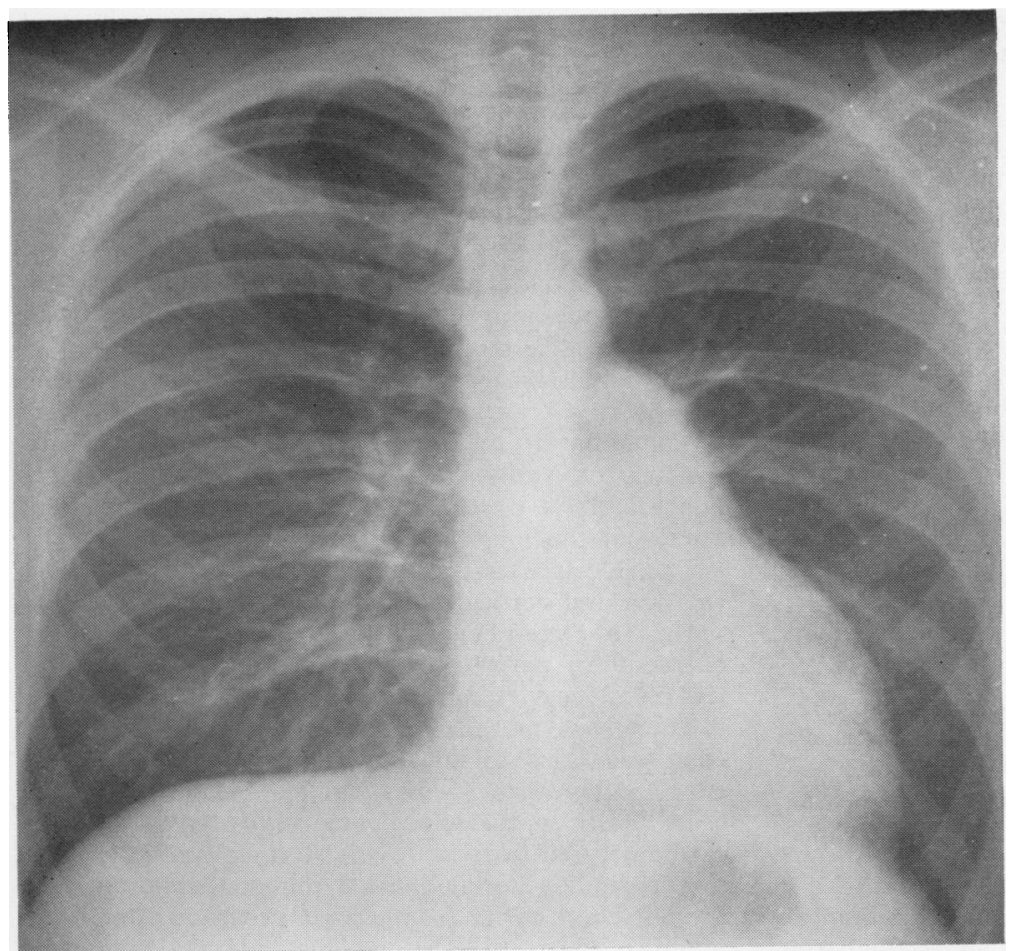

FIG. 1. Case 1. Postero-anterior chest radiograph showing displacement of the heart to the left and sharp outline of the right side of the thoracic vertebral bodies.

FIG. 2. Case 1. View at left postero-lateral thoracotomy for occlusion of persistent ductus arteriosus showing the congenital complete absence of the left side of the pericardium.

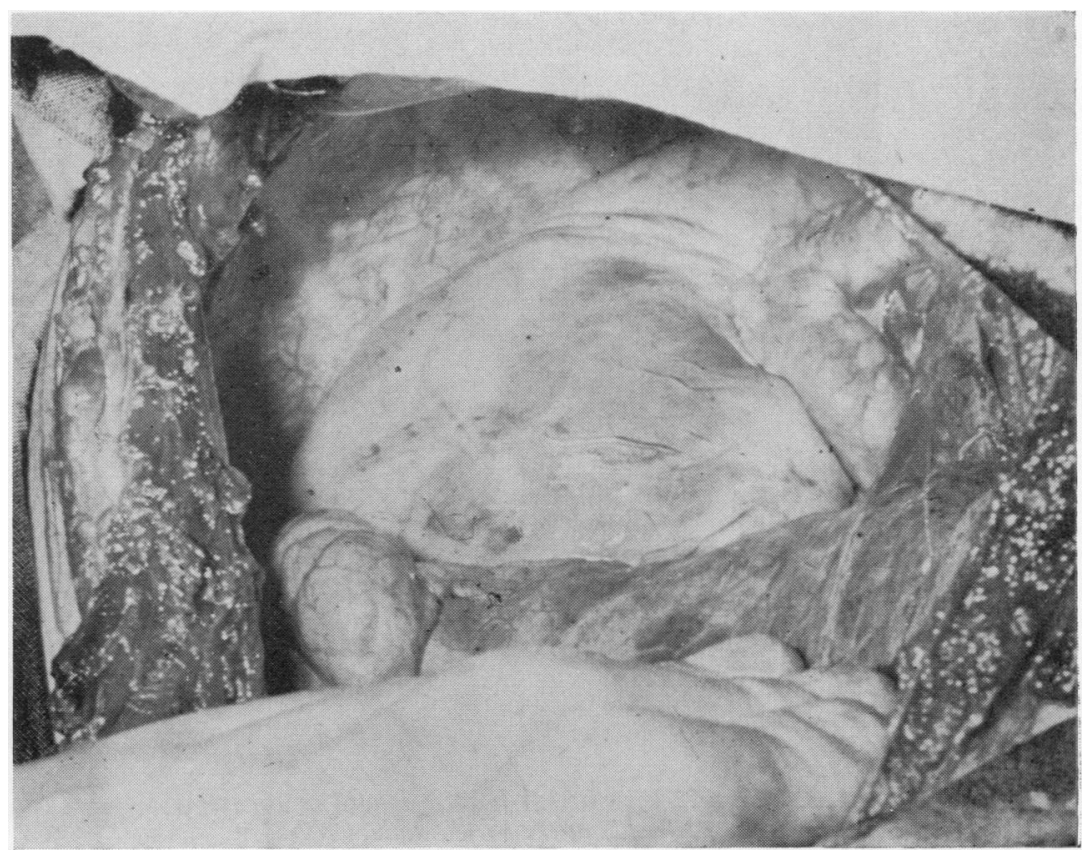


arteriosus. Retraction cf the lung revealed total absence of the left pericardium. The whole of the left side of the heart, the left ventricle, atrium, and great vessels were in free communication with the left pleural cavity. The left phrenic nerve passed down the anterior mediastinum medial to the internal mammary vessels till finally it entered the diaphragm in relation to its sternal origin. The left vagus nerve, having crossed the arch of the aorta, passed down the posterior mediastinum with the oesophagus. Palpation revealed an intact right half of the pericardium, adherent in front to the sternum, and behind fusing with the peri-aortic mediastinal pleura (Fig. 2). The typical ductal thrill was localized to one point of the left pulmonary artery.

Procedure The mediastinal pleura in the region of the ductus was vertically incised lateral to the aorta, lifted up as a flap and swung medially, carrying with it the left vagus nerve and its recurrent laryngeal branch. The patent ductus arteriosus, measuring $1 \mathrm{~cm}$. long by $0.4 \mathrm{~cm}$. wide, was next isolated, encircled, and occluded with three No. 18 linen ligatures. The pre-occlusion pressure in the aorta was $120 / 55 \mathrm{~mm}$. $\mathrm{Hg}$. The post-occlusion pressure was $120 / 80 \mathrm{~mm}$. Hg. The chest wall was closed by standard technique. The patient made an uninterrupted convalescence and was discharged 10 days later.

Follow-up $\mathrm{He}$ has been seen regularly at six-monthly intervals since then. He remains fit, active, and well.
Check chest films show no change in the position of the heart, which is in no way embarrassed by the tota lack of the left pericardium. The right sides of the thoracic vertebral bodies are clearly seen, without any overlying cardiac shadow.

He has since been accepted as a first-class life risk by the insurance company.

CASE 2 Mrs. M.E.H. (106705), aged 65 years, was admitted on 11 December 1967 for the further treat ment of squamous-cell carcinoma of the cardia, asso ciated with one month of progressive dysphagia.

The admission chest film was reported as showing no heart abnormality. However, subsequent reos examination of these films showed displacement of the heart towards the left with clear display of theo hilar shadows of the right lung and also the righe border of the thoracic vertebral column.

The electrocardiogram showed strong clockwise $Z$ rotation and vertical electrical axis.

On 18 December she had a left postero-latera thoracotomy performed. The findings on opening the left pleural cavity were again unusual (Fig. 3).

There was no left pericardium. The anterior free edge was fused to the diaphragm, the posterior frees edge to the aortic mediastinal pleura. The heart lay quite freely in the left pleural cavity with the pericardium fused in front to the sternum and behind to the descending aorta. The left phrenic nerve, entering the left chest cavity from above, proceeded down the anterior chest wall behind the internal mammaryo

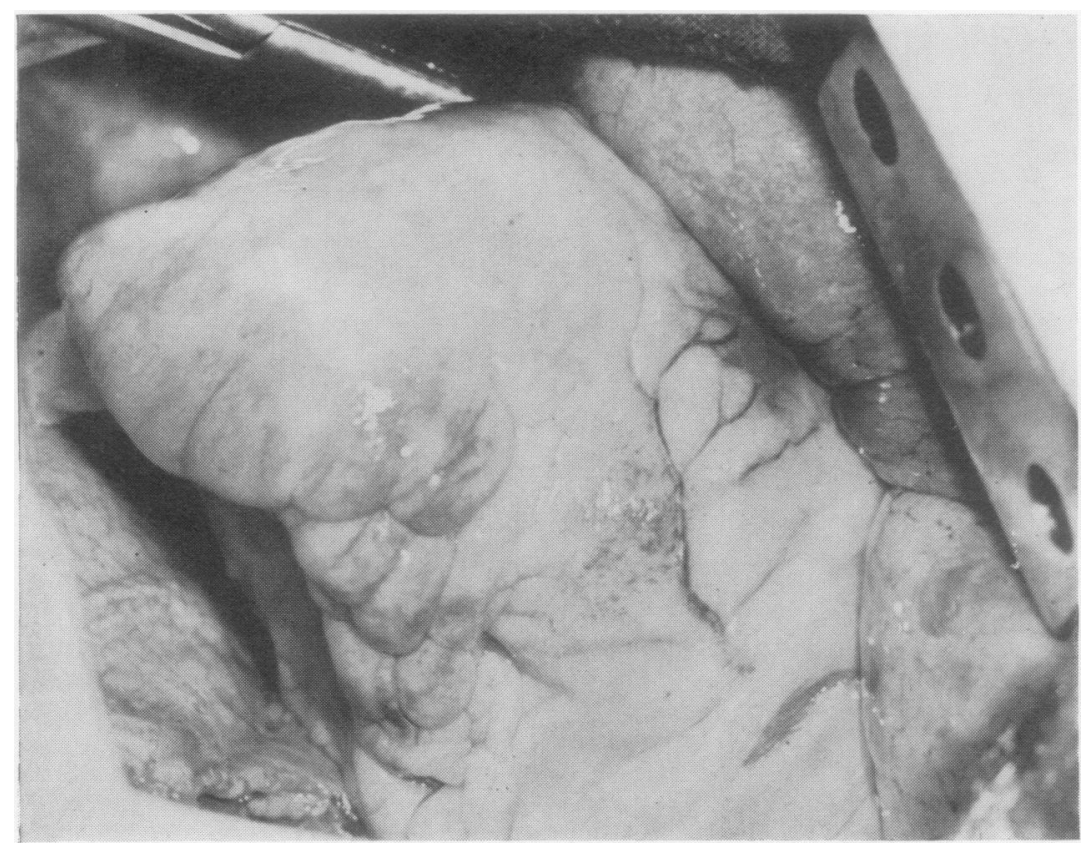

FIG. 3. Case 2. View at left postero-lateral thoracotomy showing congenital complete absence of the left side of the pericardium. 
artery to the diaphragm. The vagus nerve, after crossing the aortic arch, proceeded down the oesophagus as usual. Palpation again showed that the right side of the pericardium was intact.

Procedure A partial oesophago-gastrectomy was performed for the carcinoma, but, because of lymph node invasion in the left gastric pedicle, this proved only palliative.

She died from further spread of the disease and multiple pulmonary infarcts on 22 January 1968.

\section{DISCUSSION}

The diagnosis of congenital absence of the pericardium can be made without thoracotomy, provided the possibility of such a lesion is kept in mind.

Embryologically, several explanations have been advanced for its occurrence. Perna (1910) suggested that it was due to a premature atrophy of the left duct of Cuvier. Patten (1946) considered it resulted from incomplete development of either the transverse septum or the pleuroperitoneal folds. Southworth and Stevenson (1938) described the embryology of the pericardium. Their own case was the first with adequate clinical data in which fluoroscopic examination findings were included.

There seems to be no satisfactory embryological explanation why more than $75 \%$ of cases occur on the left-hand side, except that the left duct of Cuvier disappears in humans (though not in sheep), whereas the right duct of Cuvier remains as the right azygos vein. It would be interesting to note if those cases of absence of the right side of the pericardium have an azygos vein remaining. If not, this would give weight to Perna's view on aetiology.

Pathologically most congenital defects are leftsided. Ellis et al. (1959) found that of 66 leftsided defects in a review of 85,48 were 'complete' and 18 'partial'. Three were right-sided, one being complete and two being foramen defects. Three cases were bilateral and included foramen type and complete defects. Just why 59 of 81 cases $(73 \%)$ occurred in males is also not explained.

I can find no instance where complete absence of one side of the pericardium has been responsible for death or in any way has interfered with the normal life expectancy of the patient. The second patient was 65 years old.

The danger is with partial defects, where an atrium or ventricle may herniate with cardiac strangulation. For example, Boxall (1887) first described the escape of the heart through an in- complete pericardial sac into the left pleural cavity, while Sunderland and Wright-Smith (1944) described how a healthy 2-year-old boy collapsed and died 14 hours after onset from herniation of the heart through a left pericardial foramen, the second such death recorded.

Whether or not herniation with cardiac strangulation occurs depends on the relative size and position of the defect. Small partial defects can be harmless, but, if larger, can permit this disastrous herniation to occur.

Cardiac strangulation has also occurred from acquired pericardial defects. For instance, Sharma, Bates, and Hurt (1959) described fatal cardiac herniation following intracardiac dissection during right pneumonectomy for lung cancer. Such a case (No. 37698) has also occurred in this hospital in a patient admitted with multiple injuries from falling from a 60 -foot $(18-\mathrm{m}$.) scaffolding. He had a fractured left os calcis, a double comminuted fracture of the left femur, a fractured pelvis, and fractured 2nd to 7 th ribs on the left side with a small haemopneumothorax. Death occurred suddenly within 48 hours. Necropsy showed dislocation and strangulation of the heart through a right pericardial rent.

Yet the acquired defect of total pericardectomy, as described and practised by Brock in his radical dissection of the mediastinum for excision of lung cancer (Brock and Whytehead, 1955), does not subsequently embarrass heart function, thus supporting the experimental evidence of Moore and Shumacker (1953).

Clinically, although the defect is mainly symptomless, patients may complain of unexplained ache in the chest or occasional pain or awareness of heart beats, especially when lying on the left side. This is particularly so when the defect is partial (Ellis et al., 1959; Hering, Wilson, and Ball, 1960 ; Tucker, Miller, and Jacoby, 1963).

Radiologically, when the defect is left-sided, there is laevoratation of the heart with undue prominence of the left pulmonary artery and the left border of the heart, giving a clear view of the right side of vertebral bodies of the thoracic spine. This, however, is not a pathognomonic radiological finding of congenital absence of the left half of the diaphragm, for such findings are also seen on an antero-posterior chest film of patients with pectus excavatum (Fig. 4). However, simple physical examination of the patient excludes that possible cause.

Baker, Schlang, and Ballenger (1965) described the clinical findings in a patient with a partial pericardial deficiency, stressing that the chest film 


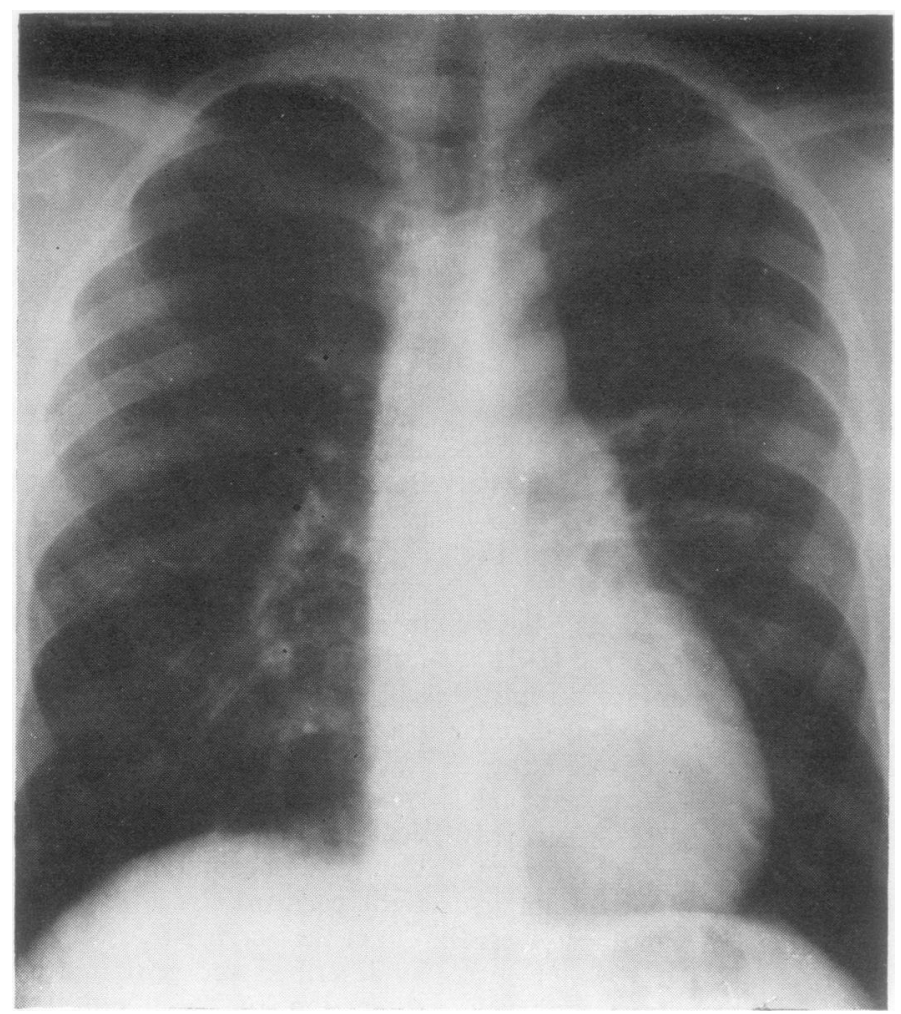

FIG. 4. Postero-anterior chest radiograph in a patient with pectus excavatum showing displacement of the heart to the left, simulating, in this view, the appearance of congenital absence of the pericardium.

revealed a prominence in the region of the main pulmonary artery. Pulmonary angiography showed that this was not the pulmonary artery but the left atrium, and a diagnostic left pneumothorax showed a pneumopericardium, establishing the diagnosis. Fluoroscopy adds little.

The diagnosis is suggested by an abnormal left hilar shadow and possibly enlargement of the pulmonary artery or left atrium, simulating pulmonary stenosis, left-to-right shunts, idiopathic pulmonary artery dilatation, primary pulmonary hypertension, mitral valve disease, as well as lung or mediastinal tumours.

It can be made by deduction from chest radiographs, as Ellis et al. (1959) have shown. It is confirmed by inducing artificial pneumothorax and taking positional films to ensure that air enters the pericardial sac from the pleural cavity. Varriale, Rossi, and Grace (1967) pointed out that the most suitable position in which to take chest films after induction of pneumothorax is either

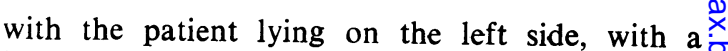
horizontally directed beam, or as a lateral film 3 . with the patient supine. As a corollary to this I suggest that the defect could be accurately seen $₹$ and confirmed by diagnostic thoracoscopy.

It is also important to distinguish between complete and partial pericardial defects. In the former, as the two cases reported here show, the heart appears displaced and rotated to the left. $\sigma$ In partial defects, the heart is in its normal site $N$ in the chest.

In partial defects this diagnosis has also been $\omega$ confirmed by angiocardiography (Hering et al., 1960 ; Kavanagh-Gray, Musgrove, and Stanwood,

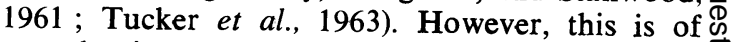
no value in total absence of the pericardium or in those partial defects where a portion of heart has 0 not herniated through the defect.

Rogge, Mishkin, and Genovese (1966) collected $\frac{\odot}{\Phi}$ 14 cases of partial defect with atrial herniation, and noted that five of these had associated con-? 
genital anomalies: bronchogenic cysts ( 2 cases); patent ductus arteriosus; tetralogy of Fallot; and atrial septal defect with atresia of the pulmonary artery orifice.

Mukerjee (1964) listed others. Case 1 reported here appears to be the second recorded as having persistent ductus arteriosus.

TREATMENT For complete defects, unless there are symptoms from pain, nothing need be done. For partial defects, surgical treatment is desirable, above all to prevent strangulation of the heart.

While Tucker et al. (1963) and Hering et al. (1960) performed the technically easy pericardiotomy, in general, closure of the defect is preferable, for not only does it prevent cardiac herniation but it also prevents spread of any later pleural infection into the pericardial cavity.

Regarding operative techniques, Jones (1955) and Baker et al. (1965) have closed partial defects with pleural flaps. Dacron or Teflon mesh is just as satisfactory, as I have confirmed experimentally in the sheep.

In conclusion, as Tucker et al. have stressed, early clinical recognition of the pericardial deficiency is of more than academic interest, since a partial defect can be responsible for sudden alarming symptoms, and complete absence allows of normal uncomplicated life expectancy, a fact that is of interest to Life Insurance Companies as well as to the patient so affected.

\section{REFERENCES}

Baker, W. P., Schlang, H. A., and Ballenger, F. P. (1965). Congenital partial absence of the pericardium. Amer. J. Cardiol., 16, 133.

Boxall, R. (1887). Incomplete pericardial sac; escape of heart into left pleural cavity. Trans. obstet. Soc. Lond., 1886, 28, 209.

Brock, R., and Whytehead, L. L. (1955). Radical pneumonectomy for bronchial carcinoma. Brit. J. Surg., 43, 8.

Columbus, R. (1559). De Re Anatomica, Book 15, p. 265. N. Beuilacquae, Venice.

D'Ath, E. F. (1969). Personal communication.

Ellis, L., Leeds, N. E., and Himmelstein, A. (1959). Congenital deficiences in the parietal pericardium. A review with 2 new cases including successful diagnosis by plain roentgenography. Amer. J. Roentgenol., 82, 125 .

Hering, A. C., Wilson, J. S., and Ball, R. E. (1960). Congenital deficiency of the pericardium. J. thorac. cardiovasc. Surg., 40, 49.

Jones, P. (1955). Developmental defects in the lungs. Thorax, 10, 205.

Kavanagh-Gray, D., Musgrove, E., and Stanwood, D. (1961). Congenital pericardial defects; report of a case. New Engl. J. Med., 265, 692.

Moore, T. C., and Shumacker, H. B., Jr. (1953). Congenital and experimentally produced pericardial defects. Angiology, 4, 1 .

Mukerjee, S. (1964). Congenital partial left pericardial defect with a bronchogenic cyst. Thorax, 19, 176 .

Patten, B. M. (1946). Human Embryology, p. 510. Churchill, London.

Perna, G. (1910). Sopra un arresto di sviluppo della sierosa pericardica nell'uomo. Anat. Anz., 35, 323.

Rogge, J. D., Mishkin, M. E., and Genovese, P. D. (1966). Congenital partial pericardial defect with herniation of the left atrial appendage. Ann. intern. Med., 64, 137.

Rusby, N. L., and Sellors, T. H. (1945). Congenital deficiency of the pericardium associated with a bronchogenic cyst. Brit. J. Surg., 32, 357.

Sharma, V. N., Bates, M., and Hurt, R. L. (1959). Herniation of the heart after intrapericardial pneumonectomy for bronchial carcinoma. Thorax, 14, 36.

Southworth, H., and Stevenson, C. S. (1938). Congenital defects of the pericardium. Arch. intern. Med., 61, 223.

Sunderland, S., and Wright-Smith, R. J. (1944). Congenital pericardial defects. Brit. Heart J., 6, 167.

Tucker, D. H., Miller, D. E., and Jacoby, W. J. (1963). Congenital partial absence of the pericardium with herniation of the left atrial appendage. Amer. J. Med., 35, 560.

Varriale, P., Rossi, P., and Grace, W. J. (1967). Congenital absence of the left pericardium and complete heart block. Report of a case. Dis. Chest, 52, 405 . 